\title{
Determination of Spatial Resolution of Positron Emission Tomograph of Clear PET-XPAD3/CT System
}

\author{
H. Olaya Dávila ${ }^{1, *}$, S. A. Martínez Ovalle ${ }^{1}$, H. Pérez ${ }^{2}$, H.Castro ${ }^{3}$ \\ ${ }^{1}$ Group of Applied Nuclear Physics and Simulation, Pedagogical and Technological University of Colombia, Colombia \\ ${ }^{2}$ ImXGam Group, Particle Physics Center of Marseille, France \\ ${ }^{3}$ Physics Department, National University of Colombia, Colombia
}

Copyright $\subset 2017$ by authors, all rights reserved. Authors agree that this article remains permanently open access under the terms of the Creative Commons Attribution License 4.0 International License

\begin{abstract}
Based on The National Electrical Manufacturers Association (NEMA), using the AMINE software to construction of sinograms and using a positron emission source of ${ }^{22} \mathrm{Na}$, were made calculations to determine the spatial resolution of a ring array system of phoswich detectors of positron emission tomograph included in the CLEAR PET-XPAD3/CT prototype for small animals, made in the laboratories of CCPM and whose project is led by the research group ImXgam. The radioactive source ${ }^{22} \mathrm{Na}$ of approximately $9 \mathrm{MBq}$ of activity, with spherical shape and diameter of $0.57 \mathrm{~mm}$ is immersed in a plexiglas disc that was located at the geometric center of tomographic system with a Field of View (FOV) of $35 \mathrm{~mm}$ in the axial and transverse directions. Displacements of radioactive source were performed on the three cartesian axes and was rebuilt a sinogram for each axis. The shape of sinogram allows describe the correct position and the maximum efficiency of each detector. Subsequently, was carried out a scanning in each one of three spatial axes taking enough distance to cover the dimensions of radioactive source. Data for each phoswich detector were recorded. The process was repeated for other axes and then radioactive source was centered with respect to the FOV and were calculated FWHM (Full Width at Half Maximum) and FWTM (Full Width at Tenth Maximum) values and performing statistics of these values with parabolic fitting, the latter setting allows to obtain parameters of spatial resolution of system.
\end{abstract}

Keywords PET, Spatial Resolution, Phoswich Detectors

\section{Introduction}

Medical imaging is a speciality that employs different methods to diagnose and to treat diseases by visualization of tissues into the human body. Currently, the radiologist is using different technologies such as: $\mathrm{X}$ ray radiography, ultrasound, Computed Tomography (CT), nuclear medicine, Positron Emission Tomography (PET) and Magnetic
Resonance Imaging (MRI). The success of medical imaging is to achieve more accurate diagnostic improving image quality with higher spatial resolution, lower noise, lower cost, decrease side effects on health and to obtain images in real time [1].

More particularly, these two techniques allow simultaneous analysis of the metabolic function of tissues and anatomical information, as the location, shape and size of the lesion. This is an important new tool to provide more accurate diagnoses of disorders that previously could not be detected early. More often, PET is used to obtain image of body's biochemistry by glucose metabolism in which it could appear and evidence in an abnormal growth of cells, such as a cancerous tumour; whereas CT may precisely locate the position of the lesion with respect to the patient anatomy.

Positron Emission Tomography is a technique that allows obtain information of metabolic function of tissues and anatomical information through metabolism of glucose or tyrosine, this technique is based on the coincidence detection of gamma rays emitted in antiparallel directions which are the result of positron annihilation with an electron. [2].

The location of injury, its contour and the spatial resolution of image depends strongly on detection system and amount of radioactive material used. However, if a large amount of radioactive material is used, the absorbed doses in the individual will increase. The new generation of scintillation crystals pixelated allows a better quality and spatial resolution of the diagnostic image. Another important point is that new detectors have short response times that reduce unwanted matches. The main objective of this work is to determine the spatial resolution of ClearPET/XPAD3-CT bimodal system.

\subsection{Theoretical Basis}

Positron Emission Tomography (PET), is an technique used in the field of nuclear medicine, where a patient or a living creature who has been previously administrated a small quantities of radioactive material through inhalation, injection or ingestion, which is metabolized in the interest 
organ or tissue. Afterwards, radioactive material disintegrates producing transitions in which a proton decays into a neutron, emitting a positron (positive beta particle) and a neutrino. The interaction between a neutrino and matter has no relevance, but the positron with energy of $1.022 \mathrm{MeV}$ interacts with an electron in the medium and annihilate producing two gamma photons of $511 \mathrm{keV}$ in antiparallel direction $\left(180^{\circ} \pm 0.5\right)$ and are responsible of the formation of the diagnostic image (Figure 1).

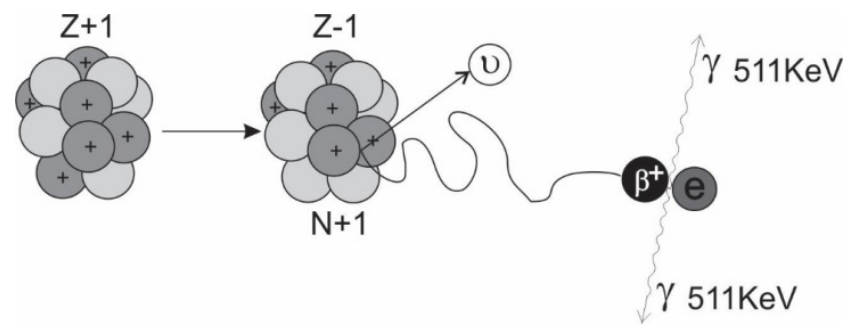

Figure 1. Annihilation and gamma photons production

The radioactive isotopes used in the PET are produced in a medical cyclotron, then taken to a radiochemistry laboratory, where they are combined with a chemical substance called tracer for latter administration. The mainly used radio-pharmaceuticals are shown in the Table 1:

Table 1. Isotopes commonly used in PET are called radiotracers. These elements are used for having a short half-life and because they can be incorporated into the human body without side effects [3]

\begin{tabular}{|c|c|c|c|}
\hline Isotope & Tracer & $\begin{array}{c}\text { Physiological } \\
\text { Process }\end{array}$ & $\begin{array}{c}\text { Main } \\
\text { Application }\end{array}$ \\
\hline${ }^{11} \mathrm{C}$ & Methionine & Protein Synthesis & Oncology \\
\hline${ }^{11} \mathrm{C}$ & Raclopride & D2 Receptor & $\begin{array}{c}\text { Movement } \\
\text { Disorder }\end{array}$ \\
\hline${ }^{13} \mathrm{~N}$ & Ammonia & Blood Perfusion & $\begin{array}{c}\text { Myocardial } \\
\text { Perfusion }\end{array}$ \\
\hline${ }^{15} \mathrm{O}$ & Water/Dioxise & Blood Perfusion & Brain Activation \\
\hline${ }^{18} \mathrm{~F}$ & Fluorine Ion & Bone Metabolism & Oncology \\
\hline${ }^{18} \mathrm{~F}$ & $\begin{array}{c}\text { Fluorine } \\
\text { deoxyglucose }\end{array}$ & $\begin{array}{c}\text { Glucose } \\
\text { Metabolism }\end{array}$ & Oncology \\
\cline { 3 - 4 } & \multirow{2}{*nyy}{} & & Neurology \\
\hline
\end{tabular}

The acquisition of the image is based upon the external detection by coincidence of the two gamma rays and a valid annihilation event requires a coincidence in the order of 12 nanoseconds of difference between the opposite detectors in the Clear PET system. Each detector generates a pulse in the temporal space when it records a photon, these pulses are combined in a coincidence circuit and are considered if both appear in a small window of time. [4]. See Figure 2.

The difference of time in the detection is known as a coincidence window and is given as $\tau=\left|\tau_{2}-\tau_{1}\right|$, where $t_{2}$ and $t_{1}$ are the times of detection for each photon.

The spatial resolution of a Positron Emission Tomograph represents its ability to distinguish between two points after image reconstruction. The measurement is performed by imaging point sources in a scattering medium. The objective of this procedure is to characterize the widths of the reconstructed image point spread functions (PSF) of a solid radioactive source of ${ }^{22} \mathrm{Na}$. The width of the PSF is defined by its Full Width at Half Maximum Amplitude (FWHM) and its Full Width at Tenth Maximum Amplitude (FWTW). The above parameters define pixel size. Finally, the intrinsic spatial resolution $R_{s}$ of the PET is performed through Derenzo formula: [5].

$$
R_{s}=a \sqrt{\frac{d^{2}}{2}+b^{2}+r^{2}+(0.0022 D)^{2}}
$$

Where $\mathbf{a}$ is an experimental factor result of the reconstruction method (which depends significantly on the filter used), $\mathbf{b}$ is the coding error of a block ( 0 when no link), $\mathbf{r}$ is the average trajectory of a positron, $\mathbf{d}$ is dimension of a pixel and $\mathbf{D}$ is diameter of the ring.

It can be observed that each parameter of the Derenzo equation contributes to increase of spatial resolution. For example, PETs for small animals have a better resolution due to reduction of ring diameter and pixel size. This is important because the anatomical structures in small animals to be studied are smaller.

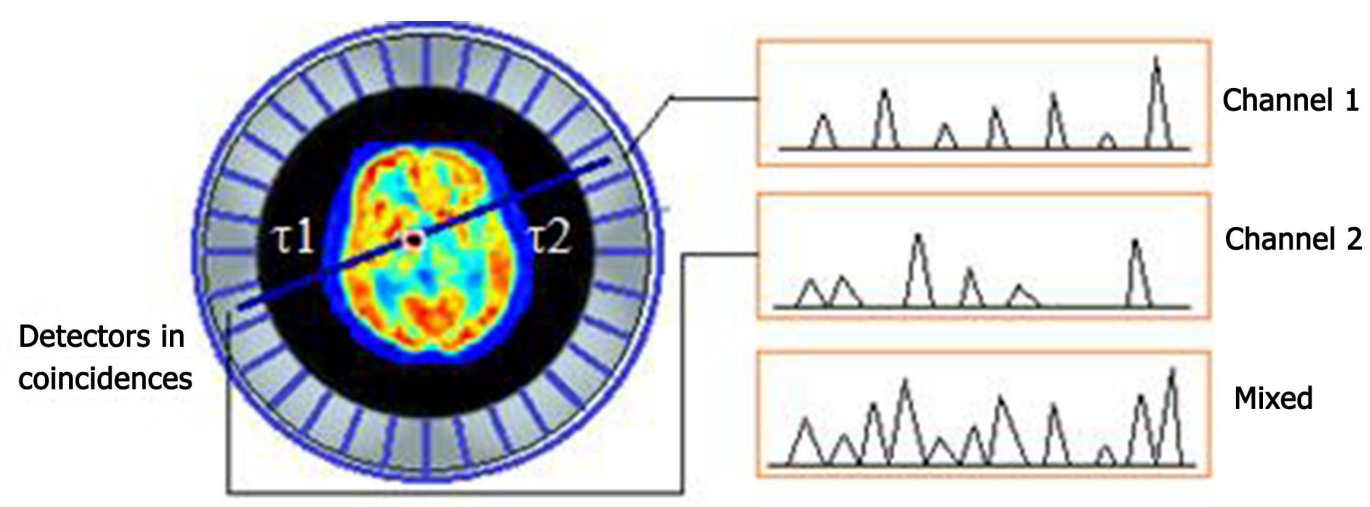

Figure 2. Coincidence of two opposite gamma photons. When a coincidence is achieved the amplitude of the pulses are added in an electric circuit. 


\section{Materials and Methods}

The ClearPET-XPAD/CT prototype bimodal for small animals was used for this work. The system is located at the ImXgam laboratories in the Centre Physique des Particules de France in Marseille, France [6]. A radioactive source of ${ }^{22} \mathrm{Na}(0.542 \mathrm{MeV})$ and $\gamma$ radiation $(1.27 \mathrm{MeV})$ of approximately $9 \mathrm{MBq}$ of activity, with spherical shape and diameter of $0.57 \mathrm{~mm}$ immersed in a plexiglas disc of $3 \mathrm{~cm}$ of diameter and was located at the geometric center of tomographic system with a Field of View (FOV) of $35 \mathrm{~mm}$ in the axial direction. The ${ }^{22} \mathrm{Na}$ is used to have energy close to its resting energy, which allows a short path before its annihilation, improving the spatial resolution. In addition, its half-life period is larger compared to radiotracers, consequently can be used as a reference radioactive.

The radioactive source is located on a support which moves through of a remote electromechanical system in axial, radial and tangential directions (3D). The geometric center of the disk with the source of ${ }^{22} \mathrm{Na}$ was fixed with a laser light and is fixed to the origin $(0,0,0)$ of tomographic system and the software of data acquisition. There was made a scanning with a length greater than the size of the disk for obtain data of intensity from the radioactive source with a Gaussian distribution. Subsequently, the source moves radially each $5 \mathrm{~mm}$ until $25 \mathrm{~mm}$ and was re-take the scanning data. For each scan was necessary to rebuild the sinogram to verify proper positioning. See Figure 4. In total five samples were performed by each displacement and for each spatial axis due to the tomographics system contains three rings of scintillation detectors phoswich: (Lutetium Oxy-ortosilicate doped with cerium $\mathrm{Lu}_{2} \mathrm{SiO}_{5}$ : Ce) and LUYAP (Lutetium
Ortoaluminate de Yttrium doped with cerium $\left.\mathrm{Lu}_{0.7} \mathrm{Y}_{0.3} \mathrm{AlO}_{3}: \mathrm{Ce}\right)$. Finally, the whole process is repeated but placing the radioactive source at a distance of $1 / 4$ of the Field of View (FOV) of tomographic system [7].

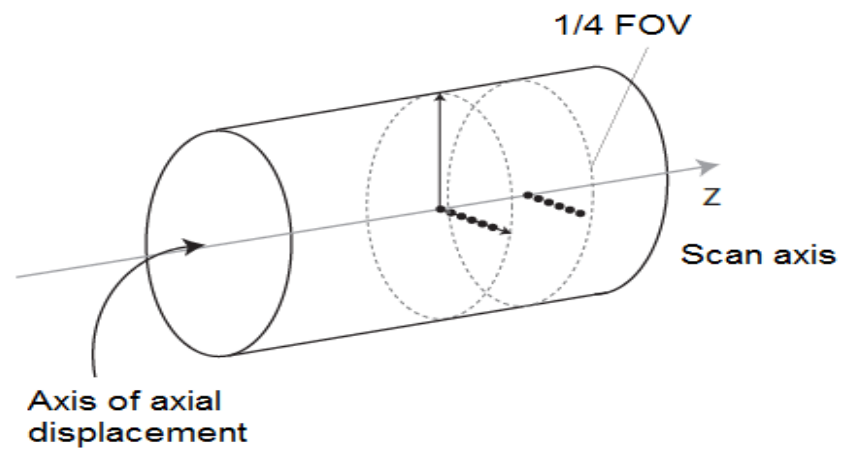

Figure 3. Scheme of positioning of ${ }^{22} \mathrm{Na}$ radioactive source. Each point represents the distance measured from the center of the axis $(5,10,15,20$ and $25 \mathrm{~mm}$ ) [8].

\section{Results}

Each data collection was performed with times about 1 minute. In each scan were obtained 130 data on average. Then, was fitted to a Gaussian distribution, where the maximum height was found by performing a second adjustment with parabolic fit in the three largest intensity data. Finally, were calculated and recorded the FWTM and FWHM values for each measurement point. See Figure 4.

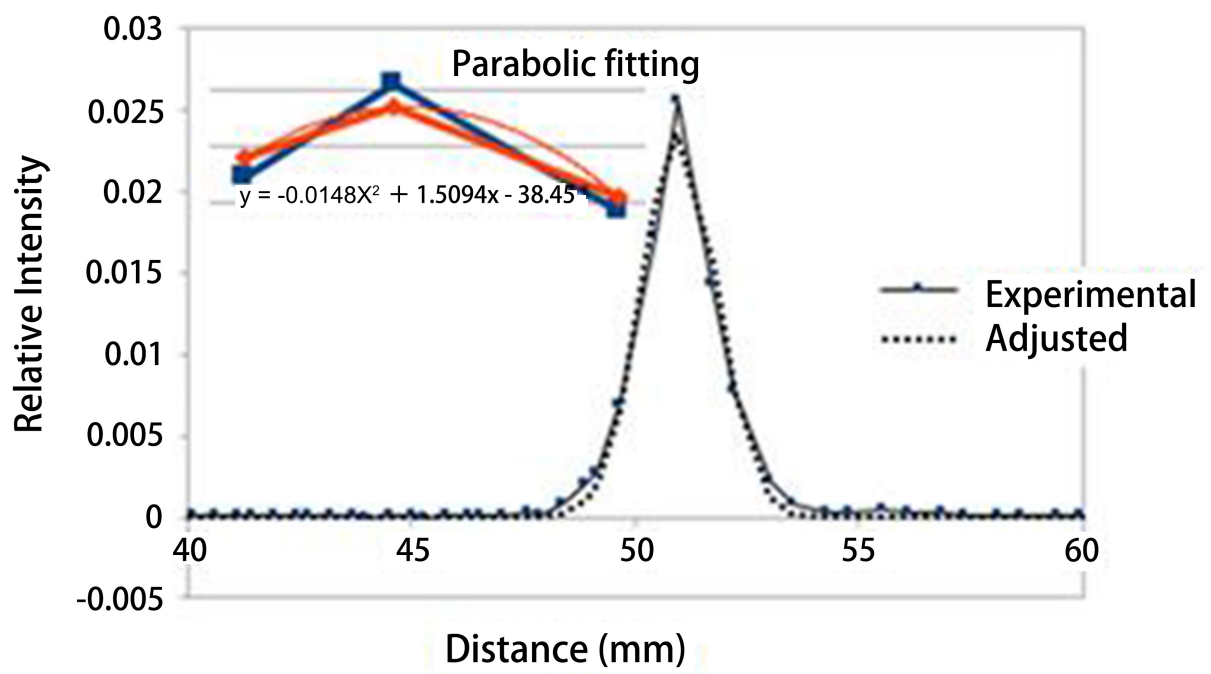

Figure 4. Spatial resolution. Adjustment of data acquired for the position $(0,0,10)$ with a scan in the radial direction. In this case FWHM=2.243 $\mathrm{mm}$ and FWTM=4.124. The parabolic fitting is only used for obtain the maximum height. 
Table 2. Spatial Resolution values calculated through parabolic fitting and weighted with those reported with Amine software. Reconstructed image pixel size (mm): 2.3; Slice thickness (mm): 0.575. At axial center.

\begin{tabular}{|c|c|c|c|c|c|c|c|c|c|}
\hline & \multicolumn{2}{|c|}{$5 \mathrm{~mm}$} & \multicolumn{2}{c|}{$10 \mathrm{~mm}$} & \multicolumn{2}{c|}{$25 \mathrm{~mm}$} \\
\hline & FWHM & FWTM & FWHM & FWTM & FWHM & FWTM & FWHM & FWTM \\
\hline Radial & 1.510 & 3.298 & 2.232 & 4.124 & 2.251 & 4.172 & 2.484 & 4.241 \\
\hline Tangential & 2.331 & 4.334 & 2.557 & 4.747 & 2.578 & 4.793 & 2.850 & 4.869 \\
\hline Axial & 1.692 & 3.261 & 1.754 & 3.584 & 1.968 & 3.554 & 2.247 & 4.072 \\
\hline \multicolumn{7}{|c|}{ At $1 / 4$ axial FOV from center } \\
\hline Radial & 2.367 & 4.320 & 2.237 & 4.124 & 2.269 & 4.128 & 2.248 & 4.122 \\
\hline Tangential & 2.550 & 4.690 & 2.588 & 4.747 & 2.566 & 4.716 & 2.601 & 4.768 \\
\hline Axial & 1.833 & 3.588 & 1.996 & 3.664 & 2.035 & 3.756 & 2.535 & 4.648 \\
\hline
\end{tabular}

In the Table 2 are shown the FWHM and FWTM for each position of the ${ }^{22} \mathrm{Na}$ at different distances for three scanning directions.

In the Figures 5 and 6 are shown values of FWHM calculated from Gaussian analysis and parabolic fitting for each position and scanning directions. Position at axial center and at $1 / 4$ of field of view. The distances $0 \mathrm{~mm}$ and 20 $\mathrm{mm}$ those are included into the figures.

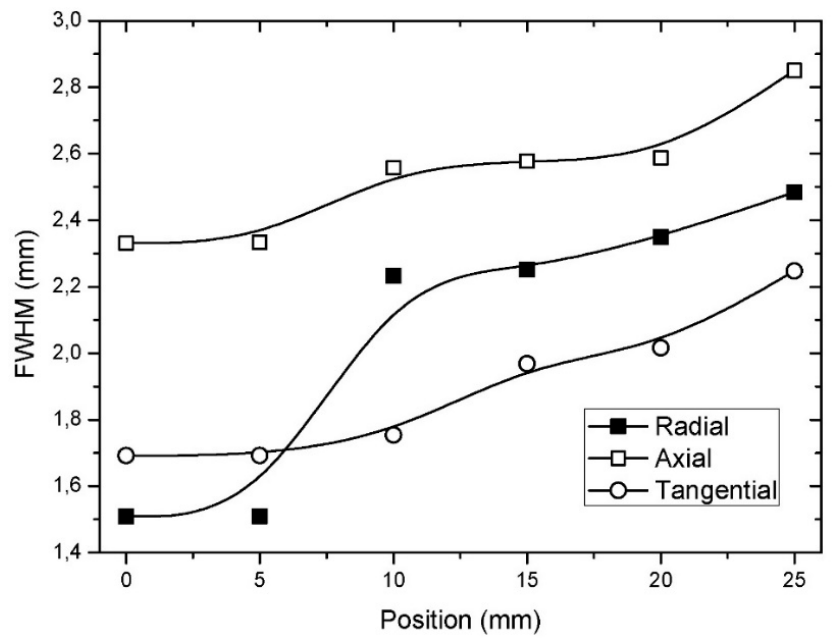

Figure 5. Spatial resolution at axial center

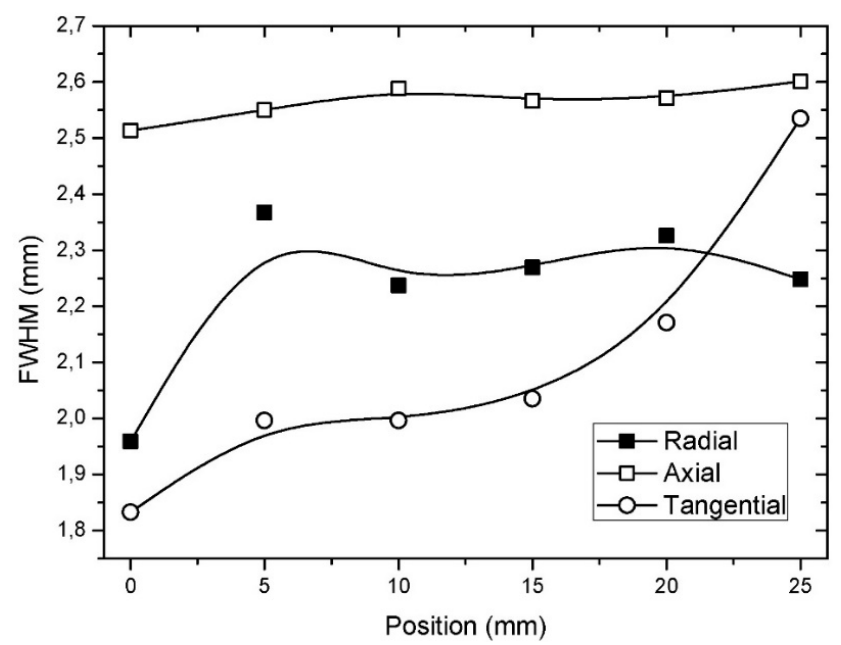

Figure 6. Spatial resolution at $1 / 4$ field of view (FOV)
Table 3. Summary of the results of the spatial resolution values in the three spatial axes is shown below:

\begin{tabular}{|c|c|}
\hline Position & Spatial Resolution $(\mathbf{m m})$ \\
\hline Radial & 2.199 \\
\hline Tangential & 2.577 \\
\hline Axial & 2.007 \\
\hline
\end{tabular}

The other method to evaluate the spatial resolution is based on geometric parameters of the bimodal system ClearPET / XPAD-CT, as shown in Table 4.

In Table 4 are shown experimental values corresponding to the intrinsic spatial resolution of equation (1) and is included the pixel size calculated through the FWHM factors.

Table 4. Parameters of intrinsic spatial resolution calculated and substituted in equation (1)

\begin{tabular}{|c|l|}
\hline $\mathrm{a}=1.2 \mathrm{~mm}$ & $\begin{array}{l}\text { Value that is calculated in the software as a result of } \\
\text { algorithm PET construction }\end{array}$ \\
\hline $\mathrm{b}=0$ & In the case of coupling a 1-1-crystal anode MaPMT \\
\hline $\mathrm{r} \cong 0.5 \mathrm{~mm}$ & $\begin{array}{l}\text { That corresponding a full width at half maximum of } \\
\text { the distribution of the mean path of positrons } \\
\text { emitted before annihilation in water by a }{ }^{22} \mathrm{Na} \\
\text { source. }\end{array}$ \\
\hline $\mathrm{d}=2.3 \mathrm{~mm}$ & Dimension of a pixel \\
\hline $\mathrm{D}=137 \mathrm{~mm}$ & Is diameter of the ring of ClearPET system \\
\hline
\end{tabular}

The result of intrinsic spatial resolution of the system ClearPET-XPAD/CT based on Derenzo formula [equation (1)] is: $R_{\mathrm{s}}=2.07 \mathrm{~mm}$

Intercomparing the results obtained in Table 3 and the calculated value Rs, the percentages of deviation between the two methods are: radial $6.2 \%$, tangential $24.5 \%$, axial $3.0 \%$.

\section{Discussion}

The methodology for obtaining the spatial resolution proposed in this paper is important to implement it within of a quality assurance program in PET, because the correct interpretation of diagnostic images metabolic depends on the quality of the image. 


\section{Conclusions}

The FWHM values obtained for Point Spread Function (PSF) method should be periodically checked and compared with the $R_{s}$ factor in order to determine errors in detection and positioning systems.

The methodology used in scanning of three spatial directions and the displacement of the radioactive source of ${ }^{22} \mathrm{Na}$ determine whether there is an abrupt change in FWHM or FWTM parameters whose origin could be the loss of efficiency of a pixel or a failure in phoswich module.

The spatial resolution will be higher in a positron emission tomograph applied in humans, because: the diameter of the ring is larger, the positron emitting isotopes such as ${ }^{18} \mathrm{~F}$ have a longer mean free path than ${ }^{22} \mathrm{Na}$ and coupling signal usually is not obtained in a multi-anode photomultiplier tube (independent signals) like ClearPET.

\section{Acknowledgements}

Special thanks to CPPM, ImXgam group by cooperation in the development of this work.

\section{REFERENCES}

[1] Badawi, R. Introduction to PET physics. Retrieved from
University of Washington Division of Nuclear Medicine website: http://depts. washington. edu/nucmed/IRL. (1999).

[2] Juweid, M. E., \& Cheson, B. D. Positron-emission tomography and assessment of cancer therapy. New England Journal of Medicine, 354(5), 496-507. (2006).

[3] Kramer, E. L., Ko, J. P., Mourtzikos, K., \& Ponzo, F. Positron Emmission Tomography Computed Tomography: A Disease-Oriented Approach. Informa Healthcare. (2008).

[4] Chantepie, B. Etude et réalisation d'une électronique rapide à bas bruit pour un détecteur de rayons $\mathrm{X}$ à pixels hybrides destiné à l'imagerie du petit animal (Doctoral dissertation, Université Aix Marseille II). (2008)

[5] Hammonet, M. Tomographie hybride simultanée TEP/TDM combinant détecteurs à pixels hybrides et modules phoswich à scintillateurs (Doctoral dissertation, Aix-Marseille Université). (2016)

[6] Nicol, S. Étude et construction d'un tomographe TEP/TDM pour petits animaux, combinant modules phoswich à scintillateurs et détecteur à pixels hybrides (Doctoral dissertation, Université de la Méditerranée-Aix-Marseille II). (2010).

[7] Dupont, M. Tomographie spectrale à comptage de photons: développement du prototype PIXSCAN et preuve de concept (Doctoral dissertation, Aix-Marseille). (2014).

[8] Luo, W., Anashkin, E., \& Matthews, C. G. Performance evaluation of a PEM scanner using the NEMA NU 4-2008 small animal PET standards. IEEE Transactions on Nuclear Science, 57(1), 94-103. (2010). 